\title{
The meandering inferior mesenteric artery
}

\author{
Melanie Strachan, MB ChB, LMCC II (Can) \\ Yousuf Vadachia, $M B C h B$ \\ Stefan Przybojewski, MB ChB, MMed \\ (Rad Diag), FCRad Diag (SA), Dip PeC (SA) \\ Department of Radiology, Stellenbosch University and \\ Tygerberg Hospital
}

A 54-year-old man presented with a longstanding history of peripheral artery disease and right leg pain for 2 weeks. An angiogram demonstrated no filling of the celiac axis or superior mesenteric artery implying stenosis/cut-off of these vessels. A meandering mesenteric artery was visible. The direction of flow in this vessel was shown by retrograde filling of the middle colic branch of the proximal superior mesenteric artery, which in turn provided collateral flow to the superior mesenteric artery, and then subsequently, collateral flow to the celiac artery through an enlarged pancreaticoduodenal artery. (Figs $1 \mathrm{a}$ and $\mathrm{b}$ ).

The digital subtraction arteriogram (DSA) imaging findings were incidental and the patient had no symptoms of mesenteric ischaemia or abdominal discomfort.

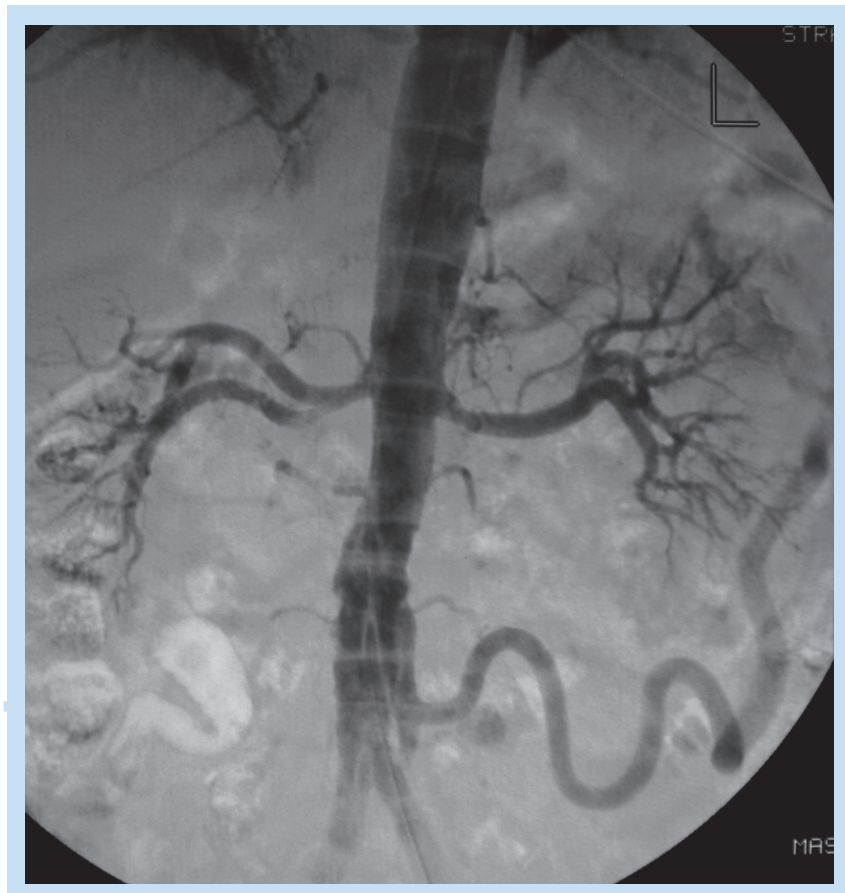

Fig. 1a. Digital subtraction arteriogram shows prograde filling of the abdominal aorta, bilateral renal arteries and inferior mesenteric artery. There is no filling of the celiac axis or superior mesenteric artery, implying stenosis or cut-off of these vessels. A meandering artery is visible.

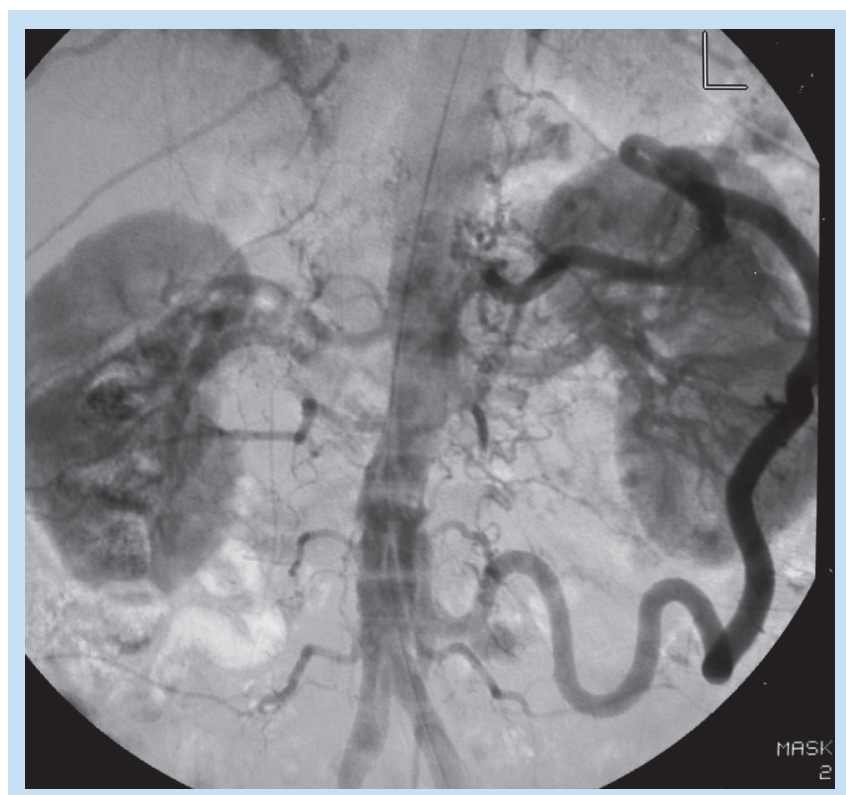

Fig. 1b. The direction of flow in the meandering mesenteric artery is shown with retrograde filling of the middle colic branch of the superior mesenteric artery. There is subsequent filling of the superior mesenteric artery by the middle colic branch.

\section{Discussion}

The meandering artery is an existing vessel, which only enlarges with abdominal arterial occlusion. The combination of superior mesenteric artery and celiac axis occlusion in an asymptomatic patient is rare and these vessels usually have retrograde filling via the meandering mesenteric artery.,

The meandering mesenteric has great surgical significance. It should therefore be noted on arteriogram and surgically preserved when performing aortic procedures, left colon resections (where the inferior mesenteric artery fills the superior mesenteric artery and/ or celiac truncus) and right and transverse colon resections (where the inferior mesenteric artery has previously been ligated during aortic surgery and is filled by the superior mesenteric artery). ${ }^{3}$

\section{Conclusion}

The meandering mesenteric artery is a rare finding with the combination of superior mesenteric artery and celiac axis occlusion. Its main function is to preserve mesenteric perfusion in patients with severe arterial disease. Therefore, it should be noted on arteriograms and surgically preserved when planning bypass surgery for peripheral arterial disease or colon resections.

\footnotetext{
1. Reiner L, Rodriques FL, Jimenez FA, Platt R. Injection studies on mesenteric artery circulation. III. Occlusion without intestinal infarction. AMA Arch Pathol 1962; 73: 461-472.

2. Kahn P, Abrahams HL. Inferior mesenteric arterial patterns: angiographic study. Radiology 1964; 82: 429-442.

3. Gourley EJ, Gering SA. The meandering mesenteric artery: a historic review and surgical implications. Dis Colon Rectum 2005; 48: 996-1000
} 